\title{
Measurement of the cumulants of net-proton multiplicity distributions by STAR
}

\author{
Roli Esha* (for the STAR Collaboration) \\ Department of Physics and Astronomy, University of California, Los Angeles \\ E-mail: roliesha@physics.ucla.edu
}

\begin{abstract}
Fluctuations and correlations of conserved quantities (baryon number, strangeness, and charge) can be used to probe phases of the strongly interacting QCD matter and the possible existence of a critical point in the phase diagram. The cumulants of the multiplicity distributions related to these conserved quantities are expected to be sensitive to possible increased fluctuations near a critical point. In this report, we present the measurements of the cumulants of net-proton multiplicity distributions from Au+Au collisions at $\sqrt{s_{N N}}=7.7,11.5,14.5,19.6,27,39,62.4$ (up to fourth order) and $200 \mathrm{GeV}$ (up to sixth order) as measured by the STAR experiment at RHIC. Multiparticle correlation functions will also be presented.

The higher order cumulants are also very sensitive to experimental artifacts that one has to deal with in the analyses of heavy-ion collision data. We demonstrate a data-driven approach to measure the cumulants of event-by-event distributions of physical variables using large samples of AMPT events. Our approach investigates the corrections for multiplicity-dependent detector response and efficiency variations. The comparison of the various correction approaches should provide important guidance towards a reliable experimental determination of the multiplicity cumulants.
\end{abstract}

Critical Point and Onset of Deconfinement

7-11 August, 2017

The Wang Center, Stony Brook University, Stony Brook, NY

${ }^{*}$ Speaker. 


\section{Introduction}

One of the main goals of the RHIC Beam Energy Scan program is to search for the QCD Critical Point (CP) and phase transition in heavy-ion collisions. Over the past years, evidence for the distinct phases of Quark Gluon Plasma (QGP) and hadron gas has been established experimentally. Lattice QCD predicts the transition from the QGP phase to the hadron gas phase to be a crossover at zero baryon density [1], while QCD-based models predict it to be a first-order phase transition for large baryon chemical potential and to end in a second-order critical point. Both theory and experiment have ruled out the existence of a critical point below baryon chemical potential $\left(\mu_{B}\right)$ values of $154 \mathrm{MeV}$ [2].

Fluctuations and correlations have been considered as sensitive observables to explore the phases of the strongly interacting QCD matter as they can provide essential information about the effective degrees of freedom. The magnitude of the fluctuation of conserved quantities (baryon number, strangeness, and charge of the system) in a grand canonical ensemble at finite temperature are distinctly different in the hadronic and the QGP phases and can be related to the susceptibility of the system. The susceptibility $(\chi)$, which is defined as the derivative of free energy density or pressure $(p)$ of a thermodynamic system at a given temperature $(T)$ with respect to the chemical potential $(\mu)$. This can be related to the cumulants $(C)$ of the event-by-event distribution of the associated conserved quantity by [3]:

$$
\chi_{q}^{(n)}=\frac{\partial^{n}\left(p / T^{4}\right)}{\partial\left(\mu_{q} / T\right)^{n}}=\frac{1}{V T^{3}} \times C_{n, q},
$$

where $V$ is the volume of the system, $n$ is the order, and $q$ is baryon number, strangeness or charge of the system. The ratios of such cumulants as experimental observables cancel the volume and temperature dependence and can be directly compared to the ratios of susceptibilities from theoretical calculations.

In order to precisely measure the event-by-event fluctuation of conserved quantities, a series of analysis techniques are applied. As no detector is perfect, an important step is particle detection efficiency correction. There could be noticeable consequences of the multiplicity-dependent behavior of particle detection efficiency on the measured higher-order cumulants [4]. In order to understand and overcome this issues, we have developed a data-driven approach for efficiency correction. This method investigates multiplicity-dependent detector response and efficiency variations.

In this report, we concentrate on the efforts at the STAR experiment to find the critical point in the QCD phase diagram at large $\mu_{B}$ and the search for experimental evidence for a smooth crossover at small $\mu_{B}$ by studying the fluctuations in net-proton (which is a proxy for net-baryon) multiplicity distributions in Au+Au collisions at $\sqrt{s_{N N}}=7.7,11.5,14.5,19.6,27,39,62.4$ (up to the fourth order) and $200 \mathrm{GeV}$ (up to the sixth order). We discuss the analysis details in Section 2, and the results in Section 3. In Section 4, we will discuss another analysis technique for efficiency correction using large samples of AMPT events. We summarize the results in Section 5.

\section{Analysis details}

STAR has a solenoidal magnetic field, full azimuthal coverage and excellent particle identification capabilities. For this analysis, we use the information from the Time Projection Chamber 
(TPC) and the Time of Flight (ToF) detector subsystems. We use the energy loss in TPC to identify protons and anti-protons for transverse momenta between 0.4 and $0.8 \mathrm{GeV} / c$ and the calculated mass from the time of flight from ToF in conjunction with the energy loss in TPC for transverse momentum between 0.8 and $2 \mathrm{GeV} / c$.

We apply the following techniques to allow for precise event-by-event fluctuation measurements and background suppression:

1. Centrality determination: The definition of collision centrality is not unique. It is usually determined by comparing experimentally measured particle multiplicity with Monte-Carlo Glauber simulations. In order to suppress auto-correlations [5], which is a background effect and can reduce the magnitude of the signal in fluctuation analyses, we exclude the corresponding protons and anti-protons from the centrality definition.

2. Centrality Bin Width Correction: The centrality bin width effect is caused by variation of volume within a wide centrality range. This results in an artificial centrality dependence for the fluctuation observable. In order to suppress volume fluctuations over wide centrality bins, the cumulants are weighted and then averaged to get the value for the given centrality. [6].

3. Statistical error estimation: Statistical errors on the cumulant of order $r\left(C_{r}\right)$ depend on the number of events $(N)$ and the width of the distribution $(\sigma)$ as :

$$
\operatorname{Error}\left(C_{r}\right) \propto \frac{\sigma^{r}}{\sqrt{N}} .
$$

These are estimated either by the Bootstrap technique, which is based on the method of resampling [7], or using the Delta Theorem, which is an analytical technique [8]. We find that these methods give similar results.

4. Detection efficiency correction: The observed event-by-event particle multiplicity distribution is a convolution of the original distribution and the efficiency response function. Efficiency correction includes the net effect of tracking efficiency, detector acceptance, decays and interaction losses. In order to correct the cumulants for efficiency, the principal idea is to express them in terms of the factorial moments [9] or factorial cumulants [10], which can be efficiency corrected assuming a Binomial response function.

\section{Results and discussions}

\subsection{Search for the QCD Critical Point}

Theory calculations show that higher-order cumulants are more sensitive to correlation lengths, thereby making them sensitive observables to critical phenomena [12]. In order to extract the multiparticle correlation functions $(\hat{\kappa})$ from the cumulants $(C)$, we use the equations in Table 1 . Here, $\langle N\rangle$ is the average number of particles over the ensemble, and the $n^{t h}$-order cumulants $\left(C_{n}\right)$ are expressed as a combination of lower order correlation functions $\left(\hat{\kappa}_{n}\right)$. The correlation functions follow the same power-law dependence on correlation lengths as the cumulants. In this analysis, we also study the energy dependence of $\hat{\kappa}_{n}$. 
Table 1: Relation between cumulants, $(C)$ and correlation functions, $(\hat{\kappa})$

$$
\begin{array}{ll}
\hat{\kappa}_{1}=C_{1} & C_{1}=\langle N\rangle \\
\hat{\kappa}_{2}=C_{2}-C_{1} & C_{2}=\langle N\rangle+\hat{\kappa}_{2} \\
\hat{\kappa}_{3}=C_{3}-3 C_{2}+2 C_{1} & C_{3}=\langle N\rangle+3 \hat{\kappa}_{2}+\hat{\kappa}_{3} \\
\hat{\kappa}_{4}=C_{4}-6 C_{3}+11 C_{2}-6 C_{1} & C_{4}=\langle N\rangle+7 \hat{\kappa}_{2}+6 \hat{\kappa}_{3}+\hat{\kappa}_{4}
\end{array}
$$

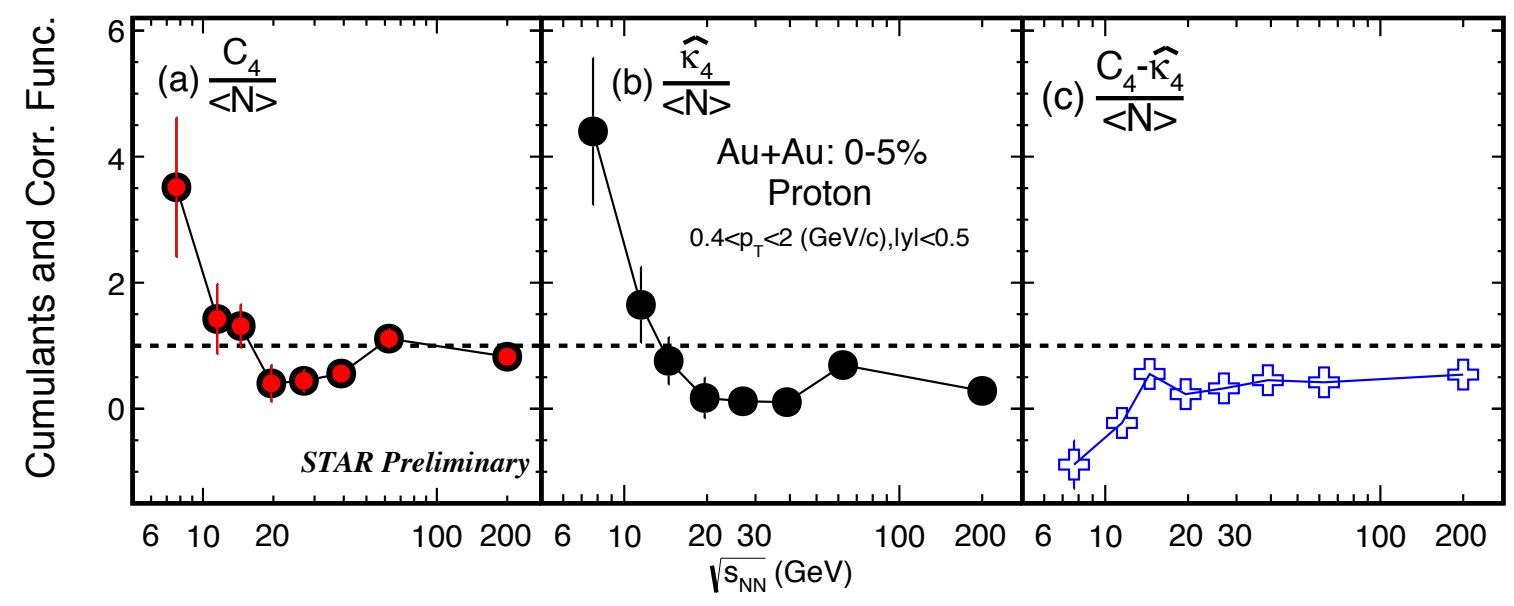

Figure 1: Energy dependence of the cumulants and correlation functions of proton multiplicity distribution in central $\mathrm{Au}+\mathrm{Au}$ collisions. The systematic uncertainties have not yet been studied.

The ratio of the fourth-order to the second-order cumulants of net-proton multiplicity distribution in most central Au+Au collisions exhibits a non-monotonic energy dependence [6]. The first panel in Fig. 1 shows the energy dependence of the normalized (by the Poisson expectation) net-proton cumulants of proton multiplicity distribution with beam energy for most central collisions [13]. We show the four-particle correlation function for protons in the second panel. It was found that the four-particle correlation functions contribute dominantly to the observed nonmonotonicity. With the removal of the fourth-order correlation function from the cumulants, only $2^{\text {nd }}$ and $3^{\text {rd }}$ correlation functions remain, see Table 1 . The results show somewhat smoother energy dependence, see Fig.1(c), and in particular no sign-change is observed.

\subsection{Constraining the QCD equation of state near $\mu_{B}=0$ region}

Lattice QCD is a well-established non-perturbative approach to solve the theory of quarks and gluons exactly from first principles. These calculations are exact at $\mu_{B}=0$, where they predict a crossover from the QGP phase to the hadron gas phase in the QCD phase diagram [1]. In order to explore phenomena at finite baryon chemical potentials, the calculations are extended using Taylor expansion about $\mu_{B}=0$. A constraint on the equation of state from Lattice QCD can be achieved by using the ratio of the sixth-order to the second-order baryon susceptibilities [14]. In addition, Lattice QCD also predicts that the ratio of the sixth-order to second-order cumulants of baryon 
number remains negative at the chiral transition temperature [15]. Combining the data from years
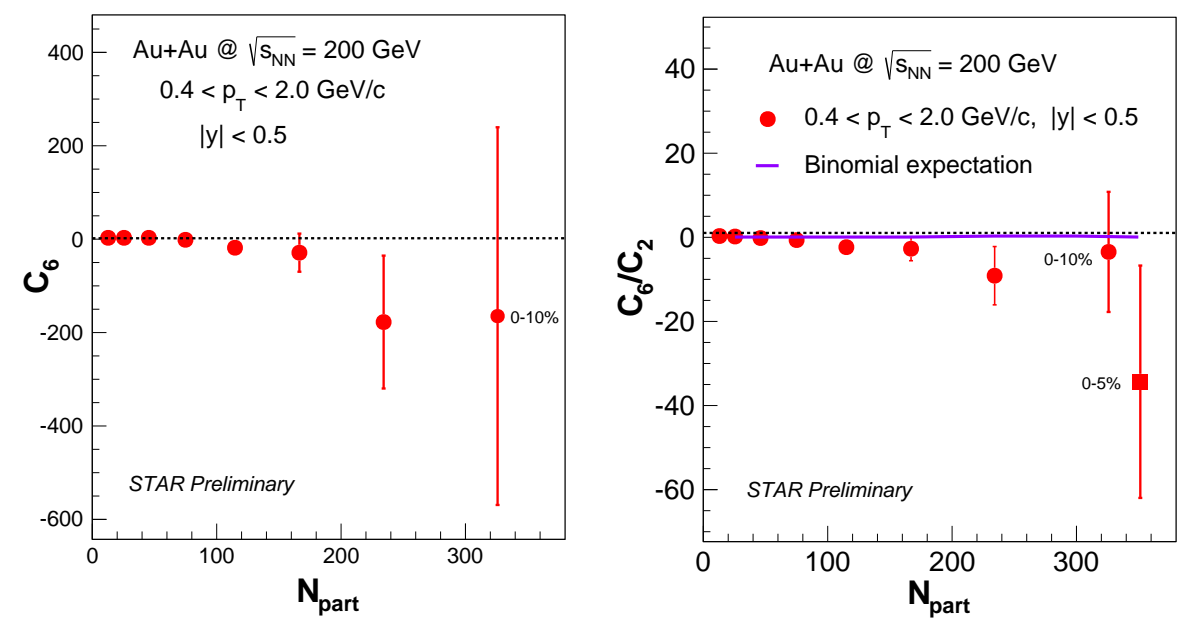

Figure 2: Centrality dependence of the sixth-order cumulants and the ratio of sixth-order to second-order cumulants of the net-proton multiplicity distribution in Au+Au collisions at $\sqrt{s_{N N}}=200 \mathrm{GeV}$. The systematic uncertainties have not yet been studied.

2010 and 2011 for $\mathrm{Au}+\mathrm{Au}$ collisions at $200 \mathrm{GeV}$, we have around $200 \mathrm{M}$ events for $0-10 \%$ central collisions (of which around $160 \mathrm{M}$ events are from year 2010) and around $650 \mathrm{M}$ events for 10 $80 \%$ central collisions. The left panel of Fig. 2 shows the values of the sixth-order cumulants, while the right panel shows the ratio of sixth-order to second-order cumulants of the net-proton multiplicity distribution as a function of number of participants for transverse momentum between 0.4 and $2 \mathrm{GeV} / c$ at midrapidity. The square markers represent the values measured using $0-5 \%$ central events from the data from the year 2010 only. For central collisions, we find the ratios of the sixth-order to the second-order cumulants of the net-proton multiplicity distributions to be negative, with large statistical uncertainties. This is consistent with the expectations from Lattice QCD [2]. The systematic uncertainties have not yet been studied.

The transverse-momentum and rapidity dependence of the ratios of the sixth-order to the second-order cumulants of the net-proton multiplicity distributions for $0-10 \%$ central collisions are shown in Fig. 3. These results are shown for data from year 2010 only.

We will continue to investigate the systematics in the transverse momentum and the rapidity dependences of the ratios of the sixth-order to the second-order cumulants. The connection with Lattice QCD calculations will require further careful investigations as we are dealing with a dynamical system.

\section{Data-driven approach for efficiency correction (simulation studies)}

The measurement of higher-order cumulants are extremely sensitive to experimental artifacts. Efficiency correction is one of the most important ingredients in order to reliably calculate the higher-order cumulants. Previous studies [4] show that there could be noticeable consequences of the multiplicity-dependent behavior of the detection efficiency on the measured higher-order 

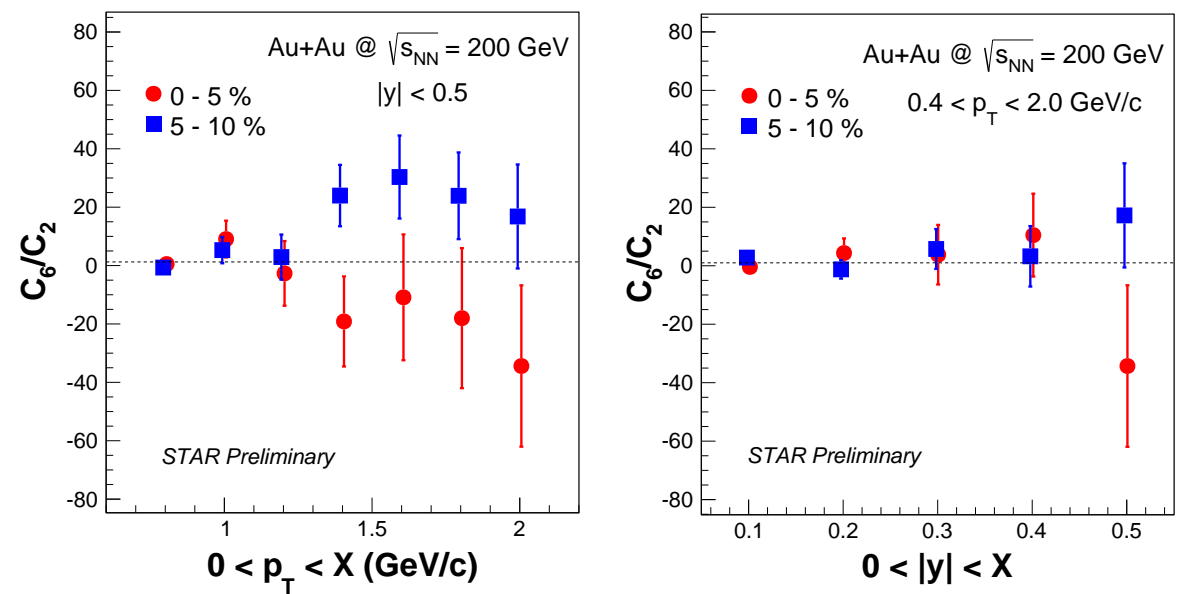

Figure 3: Acceptance dependence of the ratios of the sixth-order to the second-order cumulants of the netproton multiplicity distribution in $\mathrm{Au}+\mathrm{Au}$ collisions at $\sqrt{s_{N N}}=200 \mathrm{GeV}$. The systematic uncertainties have not yet been studied.

cumulants. In order to understand these, we have explored a data-driven approach to measure the cumulants of event-by-event distributions of physical variables using large samples of AMPT events. Comparing different methods would enable us to better understand the cumulants of these multiplicity distributions.

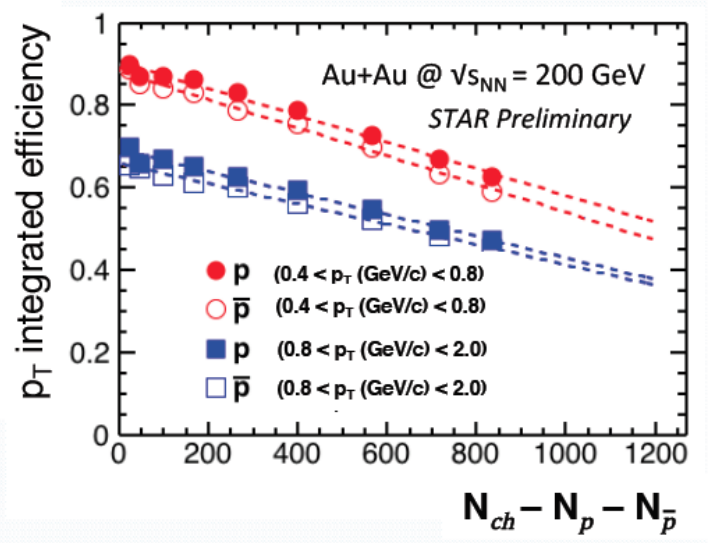

Figure 4: Efficiency of protons and anti-protons as a function of charged particle multiplicity (excluding protons and anti-protons) in $\mathrm{Au}+\mathrm{Au}$ collisions at $\sqrt{s_{N N}}=200 \mathrm{GeV}$

Figure 4 shows the efficiency of protons with the closed symbols and anti-protons in open markers integrated over transverse momentum as a function of charged particle multiplicity (excluding protons and anti-protons) in $\mathrm{Au}+\mathrm{Au}$ collisions at $\sqrt{s_{N N}}=200 \mathrm{GeV}$. The red markers are for transverse momentum between 0.4 to $0.8 \mathrm{GeV} / c$ (TPC only), while the blue markers are for transverse momentum between 0.4 to $2.0 \mathrm{GeV} / c$ (TPC + ToF). We find that the detection efficiency decreases with particle multiplicity with the slope of the order of $10^{-3}$. In order to deal with such 
multiplicity-dependent efficiency, we have explored the method described in Section 4.1.

\subsection{Methodology}

The measured distribution is a convolution of the original produced particle distribution and the detector response function. The detector response function is used to extract the original produced particle distribution. The detector response function is obtained with embedding, where a few Monte Carlo tracks are embedded within a real experimental event and passed through the entire reconstruction chain. By studying the response of the reconstruction framework on these embedded Monte Carlo tracks, we can obtain the estimate of the detector response function.

The ingredients for the implementation of the data-driven technique are the correlation histogram and the response matrix. The correlation histogram contains the number correlation between the measured protons and anti-protons event-by-event. We can obtain the response matrix using the information from the embedded Monte Carlo tracks, which gives the distribution of the number of produced particles, for every given number of measured particles.

In order to obtain the cumulants of the multiplicity distributions, the event is sampled from the correlation histogram, that is, the number of measured protons and anti-protons in event is obtained. The number of protons and anti-protons is then corrected using the respective response matrices to get the produced number of protons and anti-protons in the event. This sampling is done for the total number of events in the true distribution. The above step is repeated $M$ number of times, where $M$ is the same number of events as in the correlation histogram (true distribution). The cumulants are then evaluated for each of these $M$ copies. The mean will be the value of the cumulant, while the width will be the corresponding uncertainty. Centrality bin width correction is not used in this approach.

\subsection{Simulation results}

For the simulations, 10 million events are used and 1000 copies are created to evaluate the cumulants and their respective errors. Three scenarios are analyzed using this data-driven method and the factorial moment method (described in Section 2) to understand the implications of various correction approaches towards a reliable measurement of the cumulants.

\section{Poisson distribution for protons and anti-protons with Binomial efficiency}

We assumed that the event-by-event distribution of protons is given by a Poisson distribution with mean 10 and anti-protons with mean 9. The efficiency for protons and anti-protons is assumed to follow Binomial distribution with detection probability being 0.8 and 0.7 respectively. We compare the results in Table 2 . We find that the results from both the data-driven method and the factorial moment method agree with the analytic values.

\section{Poisson distribution for protons and anti-protons with multiplicity-dependent efficiency}

We assumed that the event-by-event distribution of protons is given by a Poisson distribution with mean 10 and anti-protons with mean 9 . The efficiency for protons is assumed to be $0.8-$ $0.0003 N_{\text {proton }}$, while for anti-protons, it is $0.7-0.0003 N_{\text {anti-proton }}$ respectively. We compare the results in Table 3. We find that the results from the data-driven method agree well with the analytic values, while the corrected cumulants obtained from the factorial moment method 
Table 2: Comparison of cumulants of net-proton multiplicity distribution, assuming a Poisson distribution for protons and anti-protons with Binomial efficiency

\begin{tabular}{|c|c|c|c|}
\hline $\begin{array}{c}\text { Cumulants for net-proton } \\
\text { distribution }\end{array}$ & $\begin{array}{c}\text { Skellam } \\
\text { (analytically) }\end{array}$ & $\begin{array}{c}\text { Efficiency corrected } \\
\text { (data-driven method) }\end{array}$ & $\begin{array}{c}\text { Efficiency corrected } \\
\text { (Factorial moment method) }\end{array}$ \\
\hline$C_{1}$ & 1 & $0.9996 \pm 0.0005$ & $1.001 \pm 0.0006$ \\
$C_{2}$ & 19 & $18.990 \pm 0.003$ & $18.990 \pm 0.004$ \\
$C_{3}$ & 1 & $1.03 \pm 0.02$ & $1.04 \pm 0.03$ \\
$C_{4}$ & 19 & $19.3 \pm 0.4$ & $18.7 \pm 0.3$ \\
\hline
\end{tabular}

starts to deviate from the analytic values for higher-order cumulants. Thus, we find that seemingly small non-Binomial effects could have noticeable consequences on higher-order cumulants.

Table 3: Comparison of cumulants of net-proton multiplicity distribution, assuming a Poisson distribution for protons and anti-protons with multiplicity-dependent efficiency

\begin{tabular}{|c|c|c|c|}
\hline $\begin{array}{c}\text { Cumulants for net-proton } \\
\text { distribution }\end{array}$ & $\begin{array}{c}\text { Skellam } \\
\text { (analytically) }\end{array}$ & $\begin{array}{c}\text { Efficiency corrected } \\
\text { (data-driven method) }\end{array}$ & $\begin{array}{c}\text { Efficiency corrected } \\
\text { (Factorial moment method) }\end{array}$ \\
\hline$C_{1}$ & 1 & $1.000 \pm 0.0004$ & $0.998 \pm 0.0006$ \\
$C_{2}$ & 19 & $19.000 \pm 0.004$ & $18.780 \pm 0.004$ \\
$C_{3}$ & 1 & $1.02 \pm 0.02$ & $1.07 \pm 0.03$ \\
$C_{4}$ & 19 & $19.1 \pm 0.3$ & $17.0 \pm 0.3$ \\
\hline
\end{tabular}

3. The AMPT model with multiplicity-dependent efficiency for 0 - $5 \%$ central Au+Au collisions at $\sqrt{s_{N N}}=200 \mathrm{GeV}$

We assumed that the event-by-event distribution of protons and anti-protons are given by the AMPT model. The efficiency for protons is assumed to be $0.8-0.0003 N_{\text {charge }}-N_{\text {proton }}-$ $N_{\text {anti-proton }}$, while for anti-protons, it is $0.7-0.0003 N_{\text {charge }}-N_{\text {proton }}-N_{\text {anti-proton }}$ respectively. The coefficient 0.0003 is the expected order of magnitude of the multiplicity dependence of efficiency in real data (Fig. 4). We compare the results in Table 4. We find that the results from the data-driven method agree well with the cumulants of the true distribution. In the 2-D response matrix situation, both protons and anti-protons are corrected simultaneously, that is, the response matrix is a two-dimensional histogram containing the information for produced number of both protons and anti-protons for every measured number of protons and anti-protons. In the 1-D response matrix approach, protons and anti-protons are corrected separately, that is, the response matrix is two one-dimensional matrices; one for protons and the other for anti-protons. The corrected cumulants obtained from the factorial moment method, however, deviate from the true values considerably. This is because the factorial moment method assumes binomial efficiency correction. Centrality bin width correction is applied to the factorial moment method. 
Table 4: Comparison of cumulants of net-proton multiplicity distribution using AMPT model with multiplicity-dependent efficiency for $0-5 \%$ central $\mathrm{Au}+\mathrm{Au}$ collisions at $\sqrt{s_{N N}}=200 \mathrm{GeV}$

\begin{tabular}{|c|c|c|c|c|}
\hline $\begin{array}{c}\text { Cumulants for } \\
\text { net-proton } \\
\text { distribution }\end{array}$ & $\begin{array}{c}\text { True } \\
\text { distribution }\end{array}$ & $\begin{array}{c}\text { Efficiency corrected } \\
\text { (2-D response } \\
\text { matrix) }\end{array}$ & $\begin{array}{c}\text { Efficiency corrected } \\
\text { (1-D response } \\
\text { matrix) }\end{array}$ & $\begin{array}{c}\text { Efficiency corrected } \\
\text { (Factorial moment } \\
\text { method) }\end{array}$ \\
\hline$C_{1}$ & $2.799 \pm 0.002$ & $2.799 \pm 0.002$ & $2.800 \pm 0.002$ & $2.550 \pm 0.001$ \\
$C_{2}$ & $31.44 \pm 0.01$ & $31.43 \pm 0.01$ & $49.78 \pm 0.02$ & $12.63 \pm 0.01$ \\
$C_{3}$ & $8.4 \pm 0.2$ & $8.4 \pm 0.1$ & $9.3 \pm 0.2$ & $2.58 \pm 0.04$ \\
$C_{4}$ & $91 \pm 1$ & $91 \pm 2$ & $89 \pm 3$ & $12.5 \pm 0.3$ \\
\hline
\end{tabular}

\section{Conclusion}

Event-by-event fluctuations in net-proton multiplicity distribution have been extensively studies at STAR with increasingly sophisticated analysis tools. We find that the non-monotonic energy dependence of the ratio of the fourth cumulant to the second cumulant of the net-proton multiplicity distribution in the most central $\mathrm{Au}+\mathrm{Au}$ collisions is closely followed by the ratio of proton cumulants. The four-particle correlations contribute dominantly to the observed non-monotonicity. With detector upgrades, more data will be collected in BES-II to provide a better insight into the possible existence and location of the QCD critical point [16].

We report the centrality, transverse momentum and rapidity dependence of the efficiencycorrected sixth-order and the ratio of sixth-order to the second-order cumulants of net-proton multiplicity distribution for Au+Au collisions at $\sqrt{s_{N N}}=200 \mathrm{GeV}$. The ratio is negative for central collisions with large statistical uncertainties. Assessment of systematic uncertainties is underway. We are also working towards combining data from high-statistics run in year 2014 and 2016 to better control statistical uncertainties.

Through Monte-Carlo simulations, we demonstrated a data-driven efficiency correction method to take into account the possible experimental effects like multiplicity-dependent detection efficiency. Implementation of this method in future STAR data analyses is under study.

\section{References}

[1] Y. Aoki et al, The order of the quantum chromodynamics transition predicted by the standard model of particle physics, Nature $\mathbf{4 4 3}$ (2006) 675.

[2] A. Bazavov et al, $Q C D$ equation of state to $O\left(\mu_{B}^{6}\right)$ from lattice $Q C D$, Phys. Rev. D 95 (2017) 054504.

[3] M. A. Stephanov, Non-Gaussian Fluctuations near the QCD Critical Point, Phys. Rev. Lett. 102 (2009) 032301.

M. Asakawa, S. Ejiri and M. Kitazawa, Third Moments of Conserved Charges as Probes of QCD Phase Structure, Phys. Rev. Lett. 103 (2009) 262301.

M. A. Stephanov, Sign of Kurtosis near the QCD Critical Point, Phys. Rev. Lett. 107 (2011) 052301.

[4] A. Bzdak, R. Holzmann and V. Koch, Multiplicity-dependent and nonbinomial efficiency corrections for particle number cumulants, Phys. Rev. C 94 (2016) 064907. 
[5] X. Luo and N. Xu, Search for the QCD Critical Point with Fluctuations of Conserved Quantities in Relativistic Heavy-Ion Collisions at RHIC : An Overview, Nucl. Sci. Tech. 28 (2017) 112.

[6] STAR Collaboration, Higher Moments of Net Proton Multiplicity Distributions at RHIC, Phys. Rev. Lett. 105 (2010) 022302.

STAR Collaboration, Beam Energy Dependence of Moments of the Net-Charge Multiplicity Distributions in Au+Au Collisions at RHIC, Phys. Rev. Lett. 113 (2014) 092301.

[7] B. Efron et al, An Introduction to Bootstrap, Chapman and Hill, 1993.

[8] X. Luo, J. Xu, B. Mohanty, N. Xu, Volume fluctuation and auto-correlation effects in the moment analysis of net-proton multiplicity distributions in heavy-ion collisions, J. Phys. G 40 (2013) 105104.

[9] A. Bzdak and V. Koch, Local efficiency corrections to higher order cumulants, Phys. Rev. C 91 (2015) 027901.

$\mathrm{X}$. Luo, Unified description of efficiency correction and error estimation for moments of conserved quantities in heavy-ion collisions, Phys. Rev. C 91 (2015) 034907.

[10] T. Nonaka, M. Kitazawa and S. Esumi, More efficient formulas for efficiency correction of cumulants and effect of using averaged efficiency, Phys. Rev C 95 (2017) 064912.

[11] B. Berdnikov, K. Rajagopal, Slowing out of equilibrium near the QCD critical point, Phys. Rev. D 61 (2000) 105017

[12] B. Ling, M. Stephanov, Acceptance dependence of fluctuation measures near the QCD critical point, Phys. Rev. C 93 (2016) 034915.

A. Bzdak, V. Koch, N. Strodthoff, Cumulants and Correlation Functions vs the QCD phase diagram, Phys. Rev. C 95 (2017) 054906.

A. Bzdak, V. Koch, V. Skokov, Correlated stopping, proton clusters and higher order proton cumulants, nucl-th/1612.05128.

[13] X. Luo, Talk at INT Workshop 2017, Seattle, US.

[14] F. Karsch and K. Redlich, Probing freeze-out conditions in heavy ion collisions with moments of charge fluctuations, Phys. Lett. B 695 (2011) 136.

[15] B. Friman, F. Karsch, K. Redlich, V. Skokov, Fluctuations as probe of the QCD phase transition and freeze-out in heavy ion collisions at LHC and RHIC, Eur. Phys. J. C 71 (2011) 1694.

[16] C. Yang (STAR Collaboration), The STAR beam energy scan phase II physics and upgrades, Nucl. Phys. A 967 (2017) 800. 